\title{
EDITORIAL
}

\section{Growing consensus in COPD?}

\author{
P. Vermeire
}

In this issue, the Journal is pleased to present to its readers the publication of a consensus statement of the European Respiratory Society (ERS) on the "Optimal assessment and management of chronic obstructive pulmonary disease (COPD)" [1]. This document was prepared by a major Task Force, established by the Executive Committee in December 1992, and it was approved in its final form by the Committee at its meeting of 13-14 May 1995. Considerable tribute should be paid to Professor Siafakas, who, since the project was started, has been the main driving force for achieving its goal. This is the first statement on the management of a major pulmonary disease, written entirely by members of the ERS, and finally published in its Journal as a consensus statement with the approval of its Executive Committee. One cannot but hope that more of these are to follow. Preparation and publication of authoritative statements should undoubtedly be a major objective of an ambitious "young" society.

Three years ago, our journal published the full version of the "International Consensus Report on Diagnosis and Management of Asthma" [2], which followed a cascade of such guidelines and statements released at a rapid rate since 1989. In an accompanying Editorial [3], I reflected on the contribution of such guidelines to improving overall management of the disease, and on the usefulness of having several consensus reports published in a short time-span. It is tempting to consider how such reflections could be adapted to the more complex entity of COPD.

As remarked in the foreword of the statement, there have been far fewer attempts to develop consensus guidelines on the management of COPD than of asthma. Hence the second question of the 1992 Editorial does not seem as relevant to COPD as it was to asthma. Despite its undoubted merits, the Canadian statement [4] did not aim to cover the subjects as comprehensively, whereas, the American Thoracic Society (ATS) statement on standards for COPD and asthma [5] was published 8 yrs earlier. However, the question has prospective relevance in that other societies are preparing, and will most likely soon publish, consensus statements on COPD management. Would it be preferable to have prolonged transatlantic international exchange of views and practices before any consensus is written and published, or might a solid international consensus on COPD develop better as the result of a variety of statements being published in succession? For asthma, it appears that the latter occurred in 1992 and that the two additional widely

Dept of Respiratory Medicine, University of Antwerp (UIA), Universiteitsplein 1, B-2610 Antwerp, Belgium. distributed statements published in the English language after that year have only perfected $[6,7]$ the consensus and made some inevitable adaptations resulting from new insights in this major field of research. The future will reveal whether this will also apply to COPD, in that independently written statements will lead to useful discussions and to a more global consensus.

Before we consider whether guidelines on COPD assessment and management, in particular, the present one can contribute to an improvement in the overall management of the disease, we need to consider whether drafting such a document is at present at all feasible. Indeed, much information is still to be obtained and many aspects still require fuller discussion and agreement. This led some prominent members of the Society to decline the invitation to join the Task Force, because they felt this effort was premature. Members of the Task Force experienced similar doubts. Therefore, like any such effort, it can be submitted to praise and criticism.

Firstly, the terminology and definitions to be used in COPD remain the subject of much confusion, well illustrated in an international survey conducted some years ago [8]. The "competing" term "chronic bronchitis" is still widely used in many countries, and can be supported by the presence of inflammatory processes in larger and smaller airways of smokers. However, when such smokers no longer cough and expectorate, the use is no longer consistent with the original Medical Research Council (MRC) definition. The consensus clearly advocates the more restricted use of the term "chronic bronchitis", but will this advice be followed? Will the view not be challenged when more is known about nonasthmatic bronchial inflammation?

"Common denominator" views have been expressed regarding investigations to be performed for initial assessment and follow-up, but are they acceptable to most respiratory physicians? Should residual volume and total lung capacity not also be measured in mild COPD, and can COPD be reliably diagnosed without these measurements? The Task Force felt it could, but practice is different in many countries.

In no other part of the statement are uncertainties as prominent as in the Treatment section. The benefits of smoking intervention are surely the best established, and their confirmation is an important achievement of the recently completed Lung Health Study [9]. Most other treatments still lack scientific support and, therefore, much of this section is based on clinical empirism. This may have led to unequal considerations being expressed regarding some treatments. As an example, long-term effects 
both of inhaled steroids and antioxidant agents in COPD have not yet been well established; therefore, on the present evidence, should recommendation against widespread use be expressed only for the latter? Surely, such sentences from the consensus should not be taken out of their full context, and the practising physician must be observant of clear benefits in individual patients, whilst waiting for the more definitive results of the major large studies that are in progress.

These are only a few critical thoughts and questions, inspired by a fresh "outside" look at the document. The main question remains as to what the consensus can contribute to improving today's management of COPD. It undoubtedly has this potential, if it can be disseminated widely among all physicians involved in the care of patients with COPD. Respiratory specialists may feel that it is beneath their level of knowledge, but this is not unexpected for a consensus document. However, they may wish to refer to it when teaching general practitioners and medical students. The nonrespiratory general physician is indeed offered valuable information. Updated background information is provided on pathology, pathophysiology and epidemiology of the disease. The complex matter of definitions has hopefully been sufficiently clarified. Appropriate use of investigations for assessment is clearly outlined, and unnecessary use is also indicated. As for asthma, goals of treatment have now also been defined, and a succinct overview is given on what is known - and not known - about the effect of most modes of treatment. The guidelines can at least now open a discussion on appropriate stepwise management; experience from daily practice will almost surely bring further refinement and simplification. Again, as in asthma, this should not be used as a recipe book without applying some common sense, and the clinician will need to maintain his clinical judgement in designing the best management for his individual COPD patient.

Widespread dissemination of the "International Consensus Report on Diagnosis and Management of Asthma" among primary health care providers in a community has recently been shown to have improved overall standards of their asthma care [10]. It is hoped that this document on COPD will achieve similar results with, however, some obvious differences; outcome parameters appear more difficult to evaluate than in asthma and present treatment leaves less room for objective improvement. Hence, more effort should be devoted to optimal assessment of quality of life in the community.

A section on areas of future research has been included in this document, as it was in the asthma consensus.
Three areas in which this should be conducted have been indicated. I wish to emphasize the need for further epidemiology research on COPD, because major information is lacking in order to achieve earlier recognition and better prevention of this disease. This is much needed, since forecasts have been issued for further increases in prevalence during the next decade, partly due to the delayed effects of the smoking epidemic and other forms of environmental air pollution.

The Editors and the Task Force emphatically welcome all further comments and questions on this consensus statement. Consensus is surely only at an intermediate stage in this complex disease, but hopefully the present effort will be appreciated.

\section{References}

1. Siafakas NM, Vermeire P, Pride NB, et al. on behalf of the Task Force. Optimal assessment and management of chronic obstructive pulmonary disease (COPD). A consensus statement of the European Respiratory Society (ERS). Eur Respir J 1995; 8: 1398-1420.

2. International Consensus Report on Diagnosis and Management of Asthma. Eur Respir J 1992; 5: 601-642.

3. Vermeire P. Growing consensus in asthma? Eur Respir $J$ 1992; 5: 509-511.

4. Chapman KR, Bowie DM, Goldstein RS, et al. Guidelines for the assessment and management of chronic obstructive pulmonary disease. Canadian Thoracic Society Workshop Group. Can Med Assoc J 1992; 147: 420-428.

5. American Thoracic Society. Standards for the diagnosis and care of patients with chronic obstructive pulmonary disease (COPD) and asthma. Am Rev Respir Dis 1987; 136: 225-244.

6. Woodhead M, (ed). Guidelines on the management of asthma. Thorax 1993; 48 (Suppl): S1-S24.

7. Global Initiative for Asthma. Global strategy for asthma management and prevention NHLBI/WHO Workshop Report. National Institutes of Health, National Heart, Lung and Blood Institute. Publication No. 95-3659. January 1995.

8. Pride N, Vermeire P, Allegra L. Diagnostic labels in chronic airflow obstruction; responses to a questionnaire with model case histories in North American and Western European countries. Eur Respir J 1989; 2: 702-709.

9. Anthonisen NR, Connett JE, Kiley JP, et al. Effects of smoking interventions and the use of an inhaled anticholinergic bronchodilator on the rate of decline of FEV1. The Lung Health Study. J Am Med Assoc 1994; 272: 1497-1505.

10. Kips JC, Joos GF, Pauwels RA. Is asthma diagnosis and treatment in general practice altered by specific teaching programmes? Am J Respir Crit Care Med 1995; 151: A613 (Abstract). 